Original Article

\title{
Association between high blood pressure, physical fitness, and fatness in adolescents
}

\author{
SERYOZHA GONTAREV ${ }^{1}$ RUZDIJA KALAC ${ }^{2}$ \\ ${ }_{1,2}$ Faculty of Physical Education, Sport, and Health, Ss. Cyril and Methodius University, Skopje, MACEDONIA \\ Published online: Octomber 30, 2016 \\ (Accepted for publication September 25, 2016) \\ DOI:10.7752/jpes.2016.s2165
}

\begin{abstract}
:
Introduction: Hypertension is a health problem that is of national importance. It is a major risk factor for the occurrence of atherosclerosis and cardiovascular, cerebrovascular and renal diseases that are leading or among the leading causes of mortality and morbitet as in ours, and in the most developed and less developed countries.Objective: The purpose of this study is to analyze the relation of cardiorespiratory fitness and obesity, blood pressure and hypertension for adolescents. The research is realized on a sample of 4051 boys and girls at the age of 11 to 14 years. The cardiorespiratory fitness was assessed by using a three minute step test, while the percentage of body fat is determined by bioelectrical impedance method. Blood pressure was measured three times at intervals of 60 seconds, and the result was the median value of the three measurements. The measurements were performed in a separate room with optimum ambient conditions. The relation between hypertension, cardiorespiratory fitness and fat is determined by correlation and multi-nominal logistic regressive analysis. Results: Boys had higher systolic pressure and lower diastolic pressure compared to girls. The low level of cardiorespiratory fitness and high percentage of body fat were independently associated with an increased risk of blood pressure and hypertension for both boys and girls. The interaction is established between the percentage of body fat and fitness. Discussion: The obtained results should be taken into account for building strategies and recommendations for improvement of lifestyle and health for adolescents

Key Words: - blood pressure; physical fitness; obesity; adolescent.
\end{abstract}

\section{Introduction}

Hypertension is a health problem that is of national importance. It is a major risk factor for the occurrence of atherosclerosis and cardiovascular, cerebrovascular and renal diseases that are leading or among the leading causes of mortality and morbitet as in ours, an in the most developed and less developed countries. Epidemiological studies show a correlation between low physical activity and/or low level of fitness and cardiovascular aliments (U.S. Department, 1997). Biological risk factors for the occurrence of cardiovascular diseases track from childhood and adolescence into adulthood (Andersen 1996; Twisk et al., 1997). The low level of fitness is associated with an increased risk of high blood pressure for middle-aged men and women (Paffenbarger et al. 1983; Blair et al., 1984). But there are many fewer researches which explain the etiological relationship between fitness and blood pressure for children and adolescents (Hagberg et al., 1983; Hagberg et al., 1994; Fraser et al., 1983).

One of the problems of the research is that the increased percentage of body fat (obesity) is associated with blood pressure and fitness. A high physical activity level may influence both fitness and body fatness. There is a consensus that aerobic training reduces blood pressure for adults, but a growing number of studies research the relation of fitness and obesity on blood pressure for children and adolescents, and there were no researches on the interaction of obesity and fitness in terms of blood pressure. Explaining the relationship between blood pressure, physical fitness and obesity is useful for building strategies and recommendations to improve the lifestyle and health of adolescents.

The purpose of this study was to analyze the relation of cardiorespiratory fitness and obesity, blood pressure, as the possible combined effects of cardiorespiratory fitness and body fat.

\section{Material \& Methods \\ Participants - Subjects}

The research is realized on a sample of 4051 adolescents with Macedonian nationality, 19 primary schools in the central and eastern part of Macedonia, 8 of which are in rural and 11 in urban areas. The sample is divided into two sub-samples according to gender and that is 2078 male respondents and 1973 female respondents. The average age of the respondents of both genders is 12.4 years. The study included all students whose parents gave consent to participate in the research that were psychologically and physically healthy, and who regularly attend classes in physical and health education. The respondents were acted in accordance with the Helsinki Declaration. 
The measurements were realized in March, April and May 2013, in standard conditions of regular school classes of physical and health education. The measurements were realized by experts in the field of kinesiology and medicine, previously trained to perform functional tests and taking anthropometric measures.

\section{Anthropometric measures and body composition}

The measurement of anthropometric measures was implemented by the recommendations $\mathrm{f}$ the IBP International Biological Program (Lohman et al., 1988). For assessment of morphological characteristics the following anthropometric measures were applied: height of the body in a standing position $(\mathrm{cm})$, weight $(\mathrm{kg})$, and body mass index (BMI).

The components of the body composition are determined by bioelectrical impedance method (measuring the electrical conductivity -Bioelektrical Impedance Analysis - BIA). The measurement is realized by Body Composition Monitor, model "OMRON - BF511", by means of which the body weight, body fat percentage and muscle mass percentage is measured. Before starting the measurement, in Body Composition Monitor were entered the parameters of gender, age and body height of the respondent. In order to ensure better accuracy of the results obtained from the assessment of body composition, before each measurement were fulfilled prerequisites recommended by ACSM (2005) and Heyward (2006).

\section{Blood pressure}

The blood pressure measurement (systolic and diastolic) is realized by experts from the medicine, doctor-specialists pediatrician fields. The measurements were performed in a separate room with optimum ambient conditions in a relaxed state of the respondent, and the relaxation is conducted at least five minutes before the measuring. The measurement was realized on forearm above the wrist on the palm, with clinically tested electronic digital device for measuring blood pressure from the company "Omron". The measurement was conducted on the left hand and before measuring care was taken that the cuff was properly inserted, the hand is at the height of the heart, and the respondent sit properly, not moving or talking. Blood pressure was measured three times at intervals of 60 seconds, and the result was the median value of the three measurements. In the age group studied, the High Blood Pressure (HBP) was regarded as the average (from three measurements) of systolic and/or diastolic pressure at the 95th percentile for age and gender, adjusted to height percentile. We adopted the methodological recommendations of the Update on the Task Force Report on High Blood Pressure in Children and Adolescent.

\section{Evaluation of Physical Fitness}

3-minute step test. Aerobic capacity is calculated using the 3-minute step test. Respondent was tasked 3 minutes to climb up and climb down from bench on high of $30.5 \mathrm{~cm}$, and that wass in four cycles (up, up, down, down) with standardized rhythm of 96 beats per minute (bmp), which enjoyed the metronome. Before beginning of the test the heart rate was measured, wherein children who are in rest condition had sub-maximal value in terms of age, they were not subjected to loading. The respondents who did not have contraindications to perform step-test immediately after the test (on cue) should sit on the bench to measure heart rate immediately after the burden, and a minute later, the stage of recovery. If the respondent felt dizziness, shortness of breath, nausea, headache or other difficulties, the test is immediately interrupted. The heart rate was measured using a monitor Polar RS800 for registration of the hearth frequency. As a result was taken the heart frequency measured one minute after the test. (Postexercise pulse rate) (Dean et al., 2011).

\section{Statistical Analysis}

The data are presented as frequencies (percentage) for categorical variables and mean (SD) for continuous variables. Gender differences in fitness, blood pressure and anthropometric characteristics were analyzed by one-way analysis of variance (ANOVA). Categorical data (weight status and proportion of hypertension ) were analyzed using the $\chi^{2}$ - test. Pearson correlation was used to analyze the relationship between blood pressure, anthropometric characteristics and fitness. The univariant and multivariant relation between hypertension, cardiorespiratory fitness and fat tissue is determined by logistic regression analysis, for the needs of the multi-nominal logistic regressive analyze the sample was divided into five categories of postexercise pulse rate and body fat, according to quintile. All the analyses were performed using the Statistical Package for Social Sciences software (SPSS, v. 22.0 for WINDOWS; SPSS Inc., Chicago, IL, USA), and values of $\mathrm{p}<0.05$ were considered statistically significant.

\section{Results}

The research was realized on a sample of 4091 respondents of which $2116(51.7 \%)$ boys and 1975 $(48.3 \%)$ girls at the age of 11 to 14 years. The average age of the respondents was $12.4 \pm 1,1$ years.

Table 1 shows the characteristics of a sample. From the review of the table 1 in which the values of the arithmetical means are shown, standard deviations and the level of statistical significance can be seen that there are statistically significant differences between the male and female gender in the variables Height, Weight, Body fat (\%), SBP (mmHg), DBP (mmHg) и Postexercise pulse rate. From the review of the obtained results, it 
can be seen that boys are taller, heavier, have a lower percentage of body fat, higher systolic pressure and lower diastolic pressure compared to girls. Statistically significant differences were found in the age and BMI variables.

The distribution of the excessive weight/obesity assessed by BMI and hypertension for adolescents in terms of gender are shown in table 1 . The table 1 analysis and summary of the $\chi^{2}$ test $\left(\chi^{2}=20.000, p=0.000\right)$ indicate that there are statistically significant differences in the nutrition level among boys and girls. The percentage values show that a higher percentage of boys are overweight/obese. Also, the statistically significant differences were established in the hypertension between boys and girls $\left(\chi^{2}=9.449, \mathrm{p}=0.002\right)$. The percentage values show that higher percentage of girls $(16,5 \%)$ have hypertension compared to boys $(14.7 \%)$.

Table 1. Description of the study population means and standard deviation (SD)

\begin{tabular}{lccccccc}
\hline & \multicolumn{2}{c}{ Boys $(\mathrm{n}=2116)$} & \multicolumn{2}{c}{ Girls $(\mathrm{n}=1975)$} & \multicolumn{2}{c}{ Total $(\mathrm{n}=4091)$} & $\begin{array}{c}\mathrm{p}- \\
\text { value }\end{array}$ \\
\cline { 2 - 8 } & Mean & SD & Mean & SD & Mean & SD \\
\hline Age (years) & 12.35 & 1.10 & 12.37 & 1.10 & 12.36 & 1.10 & 0.530 \\
Height (cm) & 157.74 & 10.94 & 156.13 & 8.08 & 156.96 & 9.70 & 0.000 \\
Weight (kg) & 52.76 & 14.71 & 51.12 & 12.07 & 51.96 & 13.52 & 0.000 \\
BMI & 20.95 & 4.17 & 20.84 & 4.07 & 20.90 & 4.12 & 0.414 \\
Body fat (\%) & 20.60 & 8.47 & 25.04 & 7.73 & 22.76 & 8.41 & 0.000 \\
SBP (mmHg) & 115.04 & 10.87 & 113.87 & 10.15 & 114.47 & 10.54 & 0.000 \\
DBP (mmHg) & 71.02 & 8.81 & 72.22 & 8.20 & 71.60 & 8.54 & 0.000 \\
Postexercise pulse rate & 112.19 & 18.57 & 130.03 & 18.64 & 120.88 & 20.63 & 0.000 \\
Proportion with systolic hypertension $\mathrm{n}(\%)$ & 207 & $10.1 \%$ & 194 & $10.0 \%$ & 401 & $10.1 \%$ & 0.958 \\
Proportion with diastolic hypertension $\mathrm{n}(\%)$ & 169 & $8.1 \%$ & 205 & $10.5 \%$ & 374 & $9.3 \%$ & 0.009 \\
Systolic and diastolic hypertension $\mathrm{n}(\%)$ & 302 & $14.7 \%$ & 355 & $18.4 \%$ & 657 & $16.5 \%$ & 0.002 \\
Proportion with overweight and obesity $\mathrm{n}(\%)$ & 386 & $39.6 \%$ & 267 & $29.7 \%$ & 653 & $34.9 \%$ & 0.000 \\
\hline
\end{tabular}

Data are shown as mean (SD). unless otherwise indicated. Sex differences were analysed by one-way analysis of variance. with sex group as fixed factors. and anthropometric or physical fitness measurements as dependent variables.

** The respondents were categorized according to the international genderand age-specific BMI $\left(\mathrm{kg} / \mathrm{m}^{2}\right)$ cut-off points (Cole et al.. 2000.2007). P $<0.010$ for difference between boys and girls (Chi-Square Tests)

Table 2 shows the correlation coefficients between the assessment of blood pressure and anthropometric indicators variables and measures to assess cardiorespiratory fitness in the total sample and separately for boys and girls. From the review of Table 2 it can be seen that for the total sample there is statistically significant correlation between systolic blood pressure, age, body weight, body height, BMI, fat percentage and postexercise pulse rate. The highest correlation with systolic pressure show the weight, BMI and body height variables. When analyzing the variables separately for boys and girls it can be seen that for both genders there is statistically significant correlation between systolic pressure, age, body weight, body height, BMI, fat percentage and post-exercise pulse rate. The highest correlation for both genders again shows the variables weight, BMI and body height.

In the total sample low statistically significant correlation with diastolic pressure show the age, weight, bogy height, body weight, BMI, fat percentage and post-exercise pulse rate variables. The highest correlation with diastolic pressure shows the post-exercise pulse rate and weight variables. When analyzing the variables separately for boys and girls it can be seen that these variables are statistically correlated with the low diastolic pressure. The highest correlation with the diastolic pressure for both genders shows the post-exercise pulse variable, which estimate the cardiorespiratory fitness.

Table 2. Relationship of systolic and diastolic blood pressure with age. height. weight. body mass index. body fat and physical fitness in Pearson correlation analysis

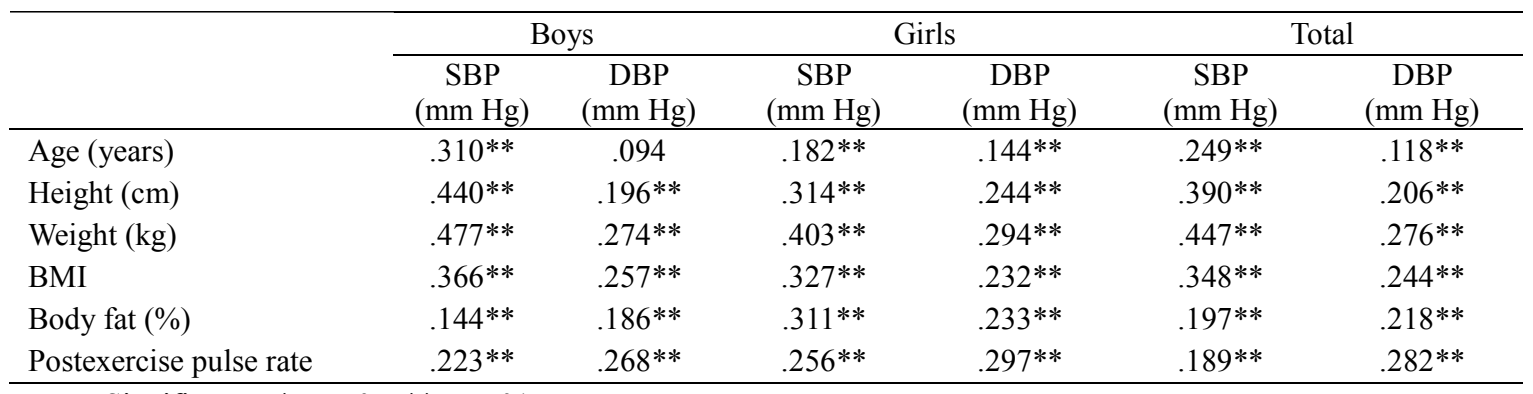


The relation between hypertension, cardiorespiratory fitness and fat tissue is determined by the multinominal logistic regressive analysis, and the results are shown in Table 3. For the purposes of the multi-nominal logistic regressive analysis the sample was divided into five categories of postexercise pulse rate and body fat, according to quintile. For boys and girls odds ratio (OR) for hypertension is calculated the univariant and multivariant between quintiles of the cardiorespiratory fitness (reference group was the highest quintile) and quintile of body fat compared with respondents who have the smallest percentage of fat (lowest quintile) (Table 3).

On the univariant level the independent $\mathrm{OR}$ for the lowest quintile of fitness compared to the highest quintile was 3.13 in boys $(\mathrm{P}=0.00)$ and 4.44 in girls $(\mathrm{P}=0.00)$, respectively. The independent $\mathrm{OR}$ for the highest quintile of body fat compared to the lowest quintile was $3.89(\mathrm{P}=0.00)$ in boys and $2.83(\mathrm{P}=0.00)$ in girls, respectively.

In order to analyze the independent association of both the cardiorespiratory fitness and the percentage of body fat with the hypertension firstly neutralization of age and body fat was done and then neutralization of age and cardiorespiratory fitness. The independent OR for the lowest quintile of fitness compared to the highest quintile was 2.18 in boys $(\mathrm{P}=0.00)$ and 3.54 in girls $(\mathrm{P}=0.00)$, respectively, after adjustment for body fat and age. The independent $\mathrm{OR}$ for the highest quintile of body fat compared to the lowest quintile was $3.04(\mathrm{P}=0.00)$ in boys and $2.28(\mathrm{P}=0.00)$ girls, respectively, after adjustment for fitness and age.

Table 3. Odds ratios and $95 \%$ confidence intervals for the relationship between hypertension and fitness as well as Body fat

\begin{tabular}{|c|c|c|c|c|c|c|c|c|}
\hline Hypertension & $\begin{array}{c}\text { Boys } \\
\text { OR }\end{array}$ & $\begin{array}{l}95 \% \\
\text { lower }\end{array}$ & $\begin{array}{l}95 \% \\
\text { upper }\end{array}$ & Pvalue & $\begin{array}{c}\text { Girls } \\
\text { OR }\end{array}$ & $\begin{array}{l}95 \% \\
\text { lower }\end{array}$ & $\begin{array}{l}95 \% \\
\text { upper }\end{array}$ & $P$ value \\
\hline \multicolumn{9}{|l|}{ Crude analyses $^{\mathrm{a}}$} \\
\hline Lowest quintile CRF & 3.13 & 2.08 & 4.71 & 0.00 & 4.44 & 2.91 & 6.79 & 0.00 \\
\hline 2nd quintile of $\mathrm{CRF}$ & 1.74 & 1.13 & 2.68 & 0.01 & 3.11 & 2.01 & 4.80 & 0.00 \\
\hline 3rd quintile of CRF & 1.21 & 0.76 & 1.93 & 0.41 & 1.73 & 1.09 & 2.74 & 0.02 \\
\hline 4th quintile of CRF & 1.05 & 0.65 & 1.69 & 0.85 & 1.75 & 1.09 & 2.79 & 0.02 \\
\hline Highest quintile of $\mathrm{CRF}$ & 1 & - & - & - & 1 & - & - & - \\
\hline Highest quintile Body fat & 3.89 & 2.58 & 5.86 & 0.00 & 2.83 & 1.95 & 4.10 & 0.00 \\
\hline 2nd highest quintile Body fat & 1.75 & 1.12 & 2.73 & 0.01 & 1.69 & 1.15 & 2.50 & 0.01 \\
\hline 3rd highest quintile Body fat & 1.39 & 0.88 & 2.21 & 0.16 & 1.22 & 0.81 & 1.84 & 0.34 \\
\hline 4th highest quintile Body fat & 1.39 & 0.88 & 2.21 & 0.16 & 1.15 & 0.76 & 1.75 & 0.50 \\
\hline $\begin{array}{l}\text { Lowest quintile Body fat } \\
\text { Multiple analyses }{ }^{\text {b }}\end{array}$ & 1 & - & - & - & 1 & - & - & - \\
\hline Lowest quintile CRF & 2.18 & 1.42 & 3.36 & 0.00 & 3.54 & 2.28 & 5.49 & 0.00 \\
\hline 2nd quintile of CRF & 1.37 & 0.88 & 2.14 & 0.16 & 2.71 & 1.74 & 4.22 & 0.00 \\
\hline 3rd quintile of CRF & 1.02 & 0.63 & 1.63 & 0.95 & 1.52 & 0.95 & 2.42 & 0.08 \\
\hline 4th quintile of CRF & 0.97 & 0.60 & 1.58 & 0.91 & 1.66 & 1.03 & 2.66 & 0.04 \\
\hline Highest quintile of CRF & 1 & - & - & - & 1 & - & - & - \\
\hline Highest quintile Body fat & 3.04 & 1.96 & 4.70 & 0.00 & 2.28 & 1.53 & 3.38 & 0.00 \\
\hline 2nd highest quintile Body fat & 1.53 & 0.96 & 2.44 & 0.07 & 1.53 & 1.01 & 2.30 & 0.04 \\
\hline 3rd highest quintile Body fat & 1.40 & 0.87 & 2.25 & 0.16 & 1.18 & 0.77 & 1.81 & 0.45 \\
\hline 4th highest quintile Body fat & 1.26 & 0.78 & 2.05 & 0.35 & 1.17 & 0.76 & 1.81 & 0.47 \\
\hline Lowest quintile Body fat & 1 & - & - & - & 1 & - & - & - \\
\hline
\end{tabular}

${ }^{\mathrm{a}}$ Univariate age-adjusted OR.

${ }^{\mathrm{b}}$ Age-adjusted OR for Body fat and fitness in the same model. Further adjusted for daily smoking. experiences with hash smoking. alcohol consumption. sweet consumption. and coffee drinking.

\section{Discussion}

The aim of this study was to determine the relation between the cardiorespiratory fitness and obesity (fatness), blood pressure and hypertension for adolescents. The advantage of this study is the relatively large number of respondents who passed the cardiorespiratory test, and they were measured as well as the body composition and the blood pressure. The study results show that there is a connection between the cardiorespiratory fitness and blood pressure /hypertension on one hand and body fats and blood pressure on the other hand.

In some previous research it is found that the relation between the cardiorespiratory fitness and blood pressure is not linear (Andersen 1994). In this sample for girls and boys it is determined almost linear relation between the blood pressure and the cardiorespiratory fitness. The respondents who belong to the first and second-fifth of the lowest cardiorespiratory fitness have significantly higher blood pressure compared to the 
remaining three-fifths. The mechanism of the relation between the blood pressure, obesity and fitness are not yet sufficiently explained and it is unknown whether these relations exist a causative relation. It is reasonable to be believed that there is a causative-consequence relation between low levels of physical activity on one hand and the increased percentage of body fat on the other hand.

However, it is also known that both properties have a strong genetic component, and on the other side the nutrition plays an important role at least in the etiology of obesity. It is often argued that the physical activity protects against obesity, due to the higher calorific consumption (Fogelholm M. \& Kukkonen-Harjula K. 2000), but it can be very simplified explanation. Theoretically it is possible as a result of decreased calorie intake to reduce weight, but maintaining it for a longer time of period without increased physical activity is rarely possible. One of the reasons for this may be the function of the enzymes in the metabolism of fats and sensitivity of metabolic hormones, especially insulin which affects the regulation of the appetite, and are not recoverable by a diet. Neither physical activity by itself is not proved successful in reducing body weight but has other benefits provided by exercise, except weight loss (Wood et al. 1991; Wood et al. 1983).

It is known that insulin sensitivity increases with aerobic. The training effect is from local character only for the trained muscles. In the study of Acts and al. it is applied an experiment which was loaded only one leg, it showed greater sensitivity on insulin, while for the untrained leg there were no changes (Dela 1996). Insulin is a hormone with many functions, and the post-serum level of the insulin is increased for people with low level of fitness as well as people with a large percentage of body fat. Besides its impact on the transport of glucose it has anabolic effect in the storage of fat in fat cells. Insulin influences the regulation of the appetite by changes in substrate levels in the blood, and the Ferranini indicates the impact on blood pressure (Ferrannini 1999). The sensitivity to insulin can be one of the key mechanisms that cause the relation between blood pressure, body fat and cardiorespiratory fitness.

Shear et al. found a positive relation between blood pressure and body fat for adolescents (Shear et al., 1987), but Stallone et al. found that the weight to BP association was due to components of body weight other than body fat (Stallones et al. 1982). There are several studies realized on children and adolescents in which has been studied the relation between blood pressure and cardiorespiratory fitness (Paffenbarger et al. 1983; Hansen et al., 1991). Hansen et al. explored the impact of the aerobic training on blood pressure for obese children (Hansen et al., 1991). After a six-month treatment in the experimental group the systolic pressure decreased by $4.9 \mathrm{mmHg}$, and the diastolic by $3.8 \mathrm{mmHg}$ compared to the controlled group.

The weight gain of $3 \mathrm{~kg}$ was similar in the two groups during the 6 months and reflected growth. Our research also confirms the results of the study Fraser et al. in which the physical fitness and blood pressure were researched in a small group of pre-adolescents and adolescents. The results of our study indicate that the change in cardiorespiratory fitness is associated with the changes in blood pressure adds to the existing evidence in adults of an association between physical fitness and blood pressure. To confirm the results, additional research is needed, especially through experimental and interventional studies. In short, the results indicate that there is a relation between the level of cardiorespiratory fitness and blood pressure and probably the change in cardiorespiratory fitness and reducing body fat is associated with blood pressure for adolescents.

\section{Conclusion}

On the basis of the obtained results it can be concluded that a higher percentage of boys are overweight/obese, have a higher systolic pressure, and lower diastolic pressure compared to girls. The low level of cardiorespiratory fitness and high percentage of body fat are independently associated with an increased risk of blood pressure and hypertension for both boys and girls. The interaction is established between the percentage of body fat and fitness. The results should be taken into account in building strategies and recommendations to improve the lifestyle and health for adolescents.

\section{Potential Conflict of Interest}

No potential conflict of interest relevant to this article was reported.

\section{Acknowledgements}

We hereby express our most heartfelt gratitude to the adolescents who took part in this study, as well as to their parents and teachers. We are deeply grateful to the Municipal Councils of Strumica and Kisela Voda, and for the funding support of the Faculty of Physical Education, Sport and Health in Skopje.

\section{References:}

ACSM (American College of Sports Medicine) Health-Related physical Fitness As sessment Manual. Balti more: Lippincott Williams and Wilkins, 2005.

Andersen, L.B. Blood pressure, physical fitness and physical activity in 17-year-old Danish adolescents. Journal of internal medicine, 236(3):323-330, 1994.

Andersen, L.B. Tracking of risk factors for coronary heart disease from adolescence to young adulthood with special emphasis on physical activity and fitness. Dan Med Bul. 43(5):407-418, 1996. 
Blair, S.N.; Goodyear, N.N.; Gibbons, L.W.; Cooper. K.H. Physical fitness and incidence of hypertension in healthy normotensive men and women. JAMA, 252(4): 487-490, 1984.

Cole, T.J.; Bellizzi, M.C.; Flegal, K.M. \& Dietz, W.H. Establishing a standard definition for child overweight and obesity worldwide: international survey. BMJ 320(7244): 1240-1243, 2000.

Cole, T.J.; Flegal, K.M.; Nicholls, D. \& Jackson, A.A. Body mass index cut offs to define thinness in children and adolescents international survey. BMJ 335(7612):194, 2007.

Dean, E. Jacks, Robert Topp, and Justin B. Moore. Prediction of $\mathrm{VO}_{2}$ Peak Using a Sub-maximal Bench Step Test in Children. Clinical Kinesiology, 65(4): 68-75, 2011.

Dela, F. On the influence of physical training on glucose homeostasis.Acta physiologica Scandinavica. Supplementum. 635: 1, 1996.

Ferrannini E. Insulin resistance and blood pressure. In: Reaven GM, Laws A., editors. Insulin resistance. The metabolic syndrome X. Contemporary endocrinology, 15. Totowa, NJ: Humana Press, 281-308, 1999 ..

Fogelholm, M.; Kukkonen-Harjula, K.Does physical activity prevent weight gain-a systematic review. Obesity reviews. 1(2): 95-111, 2000.

Fraser, G.E.; Phillips, R.L. \& Harris R. Physical fitness and blood pressure in schoolchildren. Circulation. 67(2): 405-412., 1983.

Hagberg, J.M.; Ehsani, A.; Goldring, D.; et al. Effect of weight training on blood pressure and hemodynamics in hypertensive adolescents. J Pediatr. 104:147-151, 1984

Hagberg, J.M.; Goldring, D.; Ehsani, A.A.; et al. Effect of exercise training on the blood pressure and hemodynamic features of hypertensive adolescents. Am J Cardiol. 52:763-768, 1983.

Hansen, H.S.; Froberg, K.; Hyldebrandt, N.; Nielsen, J.R. A controlled study of eight months of physical training and reduction of blood pressure in children: the Odense schoolchild study. BMJ. 303(6804): 682-685, 1991.

Heyward VH. Advanced fitness assessment and exercise prescription 5-th edition. Champaign: Human Kinetics Publishers, 2006.

Lohman, T.G.; Roche, A.F. \& Martorell, R. Anthropometric standardization reference manual. Human Kinetics Books, 1988.

Paffenbarger, R.S.; Wing, A.L.; Hyde, R.T.; Jung, D.L. Physical activity and incidence of hypertension in college alumni. Am J Epidemiol. 117(3): 245-257, 1983.

Shear, C.L.; Freedman, D.S; Burke, G.L.; Harsha, D.W.; Berenson, G.S. Body fat patterning and blood pressure in children and young adults. The Bogalusa Heart Study, Hypertension. 9(3): 236-244, 1987.

Stallones, L.; Mueller, W.H.; Christensen, B.L. Blood pressure, fatness, and fat patterning among USA adolescents from two ethnic groups. Hypertension, 4(4): 483-486, 1982.

Task Force on Blood Pressure Control in Children. Report of the Task Force on Blood Pressure Control in Children. Pediatrics. 59(5 2 suppl): S797-820, 1977.

Twisk, J.W.R.; Kemper, H.C.G.; Mechelen, W,v.; Post, G.B. Tracking of risk factors for coronary heart disease over a 14-year period: a comparison between lifestyle and biologic risk factors with data from the Am- sterdam Growth and Health Study. Am J Epidemiol. 145(10): 888-898, 1997.

U.S. Department of Health and Human Services. Physical activity and health: a report of the Surgeon General. U.S. Department of Health and Human Services, 1997. Atlanta: Centers for Disease Control and Prevention, National Center for Chronic Disease Prevention and Health Promotion.

Wood, P.D.; Haskell, W.L.; Blair, S.N.; Williams, P.T.; Krauss, R.M.; Lindgren, F.T.; Albers, J.J.; Ho, P.H.; Farquhar, J.W. Increased exercise level and plasma lipoprotein concentrations: a one year, randomized, controlled study in sedentary middle-aged men. Metabolism. 32(1): 31-39, 1983.

Wood, P.D.; Stefanick, M.L.; Williams, P.T.; Haskell, W.L. The effects on plasma lipoproteins of a prudent weight-reducing diet, with or with- out exercise, in overweight men and women. New England Journal of Medicine,325(7): 461-466, 1991. 